\title{
Cuantificación del deterioro funcional durante seis meses en pacientes renales en estadio terminal
}

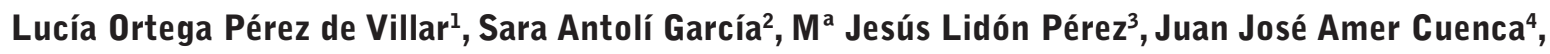 \\ Javier Martínez Gramage ${ }^{5}$, Eva Segura Ortí ${ }^{6}$
}

1,2,3,4,5,6Fisioterapeuta. Departamento de Fisioterapia Universidad CEU Cardenal Herrera. ${ }^{3}$ Supervisora de Enfermería, Hospital Universitario Doctor Peset. Valencia. España

\section{Resumen}

Introducción: Los pacientes en tratamiento de diálisis presentan una disminución de la función física, sin embargo, no hay estudios que demuestren el ritmo al que se produce este deterioro funcional. El objetivo principal es cuantificar el deterioro funcional de los pacientes en tratamiento de diálisis durante seis meses.

Métodos: 43 pacientes en tratamiento de diálisis de dos centros de Valencia (España) (edad 61.3 (14.7) años) fueron analizados después de 6 meses. Los participantes completaron el Short Physical Performance Battery, equilibrio monopodal, el Timed Up and Go Test, Sit to stand to Sit Test 10 y 60, dinamometría de mano; elevación de talón y 6 minutos marcha. Además se recogieron datos de las historias clínicas y de las analíticas.

Resultados: Tres de las pruebas mejoraron significativamente tras el periodo de 6 meses de observación: el Sit To Stand 10, la dinamometría de la mano derecha y la dinamometría de la mano izquierda, pero sin alcanzar un cambio clínico relevante. Por otro lado, otras medidas no obtuvieron un deterioro significativo como fueron el Short Physical Performance Battery, el Sit to Stand 60, la elevación del talón izquierdo y el 6 minutos marcha.

Conclusiones: Después de 6 meses, en ninguna de las pruebas de capacidad funcional se observa un de-

\section{Correspondencia:}

Departamento de Fisioterapia,

Universidad CEU Cardenal Herrera

Avda. Seminario, s/n. 46113 Moncada. Valencia. España

lucia.ortega@uchceu.es terioro significativo. Se recomienda a los centros de hemodiálisis realizar un seguimiento de capacidad funcional anualmente, ya que con un periodo de 6 meses no se encuentran cambios significativos.

\section{PALABRAS CLAVE}

- INSUFICIENCIA RENAL CRÓNICA

- CAPACIDAD FUNCIONAL

- DETERIORO FUNCIONAL

Quantification of functional impairment for six months in hemodialysis patients

\section{Abstract}

Introduction: Patients in dialysis treatment present a decrease in physical function. However, we cannot find in the literature the rhythm of the functional deterioration. The main aim is quantify the functional deterioration in patients undergoing haemodialysis during six months period.

Methods: 43 patients in dialysis treatment from two centres from Valencia (Spain) (age 61.3 (14.7) years old) were recruited and monitored after the 6 months period. Study participants performed the Short Physical Performance Battery, one leg stand, TUG, Sit to Stand 10 and 60, handgrip dynamometry, the one leg heel rise and the 6 minutes walking time.

Outcomes: Three tests improve significantly: the Sit to Stand 10, the right and the left Handgrip Dynamometry. Nevertheless, we cannot observe a significant deterioration as the Short Physical 
Performance Battery, the Sit to Stand 60, the one leg heel rise in the left leg and the 6 minutes walking time.

Conclusions: After 6 months, we have not observed a significant deterioration in the tests. We recommended the different units to follow the functional capacity of the patients yearly, because with a period of 6 months we have not find significantly changes.

\section{KEYWORDS}

- CHRONIC KIDNEY DISEASE

- FUNCTIONAL CAPACITY

- FUNCTIONAL DETERIORATION

\section{Introducción}

La insuficiencia renal crónica (IRC) en estadio terminal presenta una prevalencia que va en aumento de año en año. En Europa, la prevalencia por millón de habitantes pasó de 779 en 2006 a 881 en 2008, mientras que en España en este mismo periodo la prevalencia presentó cifras superiores, pasando de 983 a 994 casos por millón de habitantes. La terapia renal substitutiva más común para las personas con este tipo de patología es la hemodiálisis (HD $)^{1,2}$

Los pacientes en tratamiento de HD presentan una disminución de la capacidad funcional ${ }^{3}$. Esta alteración funcional ya es evidente en estadíos previos de insuficiencia renal $l^{4,5}$, aunque no se conoce si está asociada a la disminución de la tasa de filtrado glomerular o a otros factores asociados con la IRC. Los pacientes en HD tienen un nivel de actividad física significativamente inferior al que presentan sus homólogos sanos ${ }^{6}$. Se desconoce a qué ritmo disminuyen la capacidad funcional y el nivel de actividad física de los sujetos en HD. Las alteraciones a nivel muscular son comunes en los pacientes con IRC terminal, entre las que se encuentran la reducción de la sección transversal, los cambios degenerativos y las alteraciones metabólicas $7,8,9$. Estas alteraciones actúan como factores limitantes de la capacidad funcional ${ }^{10}$. Además, es común encontrar en HD un bajo nivel de calidad de vida relacionada con la salud, asociada frecuentemente a la presencia de depresión y ansiedad $11,12,13,14,15$

El objetivo principal de este estudio es cuantificar el deterioro funcional de los pacientes en tratamiento de hemodiálisis durante seis meses.

\section{Material y Métodos}

\section{Sujetos}

Se incluyeron cuarenta y tres pacientes ( 28 hombres y 15 mujeres) con insuficiencia renal crónica en estadio terminal de la unidad de diálisis al Hospital Virgen del Consuelo de Valencia y del Hospital Universitario Doctor Peset (España) para participar en el estudio entre octubre de 2014 y enero de 2015.

Los criterios de inclusión fueron: estar al menos 3 meses en tratamiento de hemodiálisis y encontrarse estable médicamente. Los criterios de exclusión fueron: (1) Infarto de miocardio en las 6 semanas previas (2) angina inestable al ejercicio o en reposo (3) amputación de miembros inferiores por encima de rodilla sin prótesis (4) enfermedad vascular cerebral (ictus, isquemias transitorias) (5) alteraciones músculo-esqueléticas o respiratorias que empeoren con las pruebas funcionales (6) imposibilidad de realizar las pruebas funcionales.

Se tomaron los siguientes datos clínicos al inicio del estudio: edad, peso seco, índice de masa corporal, tiempo en HD, horas de HD a la semana, índice de comorbilidad de Charlson.

La capacidad física funcional se midió al inicio y a los 6 meses sin realizar ningún tipo de intervención, los participantes tenían que realizar su vida normal.

El estudio fue aprobado por el Comité de Ética de la Universidad CEU Cardenal Herrera y por el Comité Ético de Investigación del Hospital Universitario Doctor Peset de Valencia, y los participantes firmaron el consentimiento informado. La investigación se realizó dentro de las pautas marcadas por la Declaración de Helsinki de 1975.

\section{Capacidad física funcional}

Las pruebas funcionales encaminadas a evaluar la condición física de los pacientes fueron el Short Physical Performance Battery (SPPB), el equilibrio monopodal, el Timed up and go (TUG), el Sit to stand to sit test 10 y 60 (STS 10 y STS 60), la dinamometría de mano, la fuerza de tríceps sural, y el 6 minutos marcha (6MWT).

La prueba Short Physical Performance Battery (SPPB) se registró el primer día antes de la sesión de HD. Consta de tres pruebas de equilibrio, con los pies juntos, en posición de semitándem y en tándem, 
y se valora si el sujeto puede mantener cada una de estas posiciones hasta un máximo de 10 segundos. La valoración del equilibrio va de 0 a 4 puntos. Incluye también una prueba de velocidad de la marcha en 4 metros a velocidad habitual. Se realizaron dos intentos cronometrados y se registró el mejor de ellos, de forma que, según los segundos empleados, se asignó una puntuación de 1 a 4 . Se valoró también la prueba STS-5, que consiste en medir el tiempo empleado en levantarse y sentarse de la silla 5 veces con los brazos cruzados en el pecho, deteniendo el cronómetro cuando el paciente alcanzaba la posición de pie en la repetición 5 . Según el tiempo empleado se asignó una puntuación de 0 a 4 puntos. Por lo tanto, la puntuación del SPPB va de 1 a 12 puntos ${ }^{16}$.

Se valoró también el equilibrio estático monopodal antes de la primera sesión semanal de HD, pidiendo a los sujetos que levantasen una pierna flexionándola de forma que se pusieses a la pata coja, con la pierna que se encontrasen más seguros. Se les pidió que aguantasen el equilibrio el mayor tiempo posible. El fisioterapeuta anotó el tiempo que el paciente aguantaba en posición de apoyo monopodal. El sujeto podía mover los brazos y flexionar la rodilla si lo necesitaba para mantener el equilibrio. El tiempo finalizaba cuando el sujeto usaba sus brazos para apoyarse, utilizaba el pie elevado para apoyarse en el suelo, cuando el pie apoyado se movía para mantener el equilibrio o cuando el tiempo llegaba a los 45 segundos. Se repitió el procedimiento 3 veces y se registró el mejor tiempo ${ }^{17}$.

La prueba 'Timed Up and Go' (TUG) ${ }^{18,19}$, también se realizó previamente a la primera sesión de hemodiálisis. Desde una silla el paciente se tenía que levantar, caminar 3 metros y volver a la posición inicial. Se repitió el procedimiento 3 veces y se registró el mejor tiempo.

La prueba de dinamometría de mano se realizó con el Dinamómetro JAMAR previamente al segundo día de la sesión semanal de HD. Según varios estudios en pacientes en diálisis, existe una correlación entre la fuerza de prensión de la mano con su estado de salud9, 20,21 . Los sujetos sentaban en una silla, con los pies apoyados en el suelo y las rodillas flexionadas $90^{\circ}$. El hombro del brazo que se estaba registrando permanecía en rotación neutra, con el codo en flexión de $90^{\circ}$ apoyado en la mesa, muñeca y antebrazo semipronados $\left(0-30^{\circ}\right)$ y una desviación cubital entre 0 y $15^{\circ}$. Se realizaron 3 repeticiones consecutivas, con un descanso de 15 segundos entre ellas, en cada uno de los miembros superiores, comenzando por el brazo dominante. Se dio ánimo verbal en las repeticiones y se registró la de valor máximo.

El segundo día de la sesión semanal de hemodiálisis también se realizaron las pruebas del STS 10 y STS 60, tal y como describen Cüska y McCarty22. La primera consiste en medir los segundos que necesita el paciente para levantarse y volver a sentarse 10 veces consecutivas lo más rápido posible, desde una posición de sentado y con los brazos cruzados en el pecho. Tras realizar las 10 repeticiones se registró el tiempo que había necesitado para realizar la prueba, así como el grado de dificultad en la Escala de Esfuerzo Percibido (EEP) (Figura 1 EEP). A continuación, tras un periodo de recuperación de 3 minutos como mínimo, se realizó el STS 60. Se explicó al paciente que la prueba consistía en realizar el máximo número de repeticiones de levantarse y volver a sentarse en un tiempo de 60 segundos. Tras la realización de la prueba se registraron las repeticiones y se anotó el grado de dificultad de la prueba según la EEP.

La prueba de la fuerza del tríceps sural o prueba de elevación de talón monopodal, se realizó también inmediatamente antes de la segunda sesión semanal de $H^{23,24,25}$. Los pacientes debían de estar descalzos. El ritmo de elevación del talón se marcó mediante un metrónomo. Antes de iniciar la prueba se pidió al paciente que mantuviese el equilibrio sobre una sola pierna, con un apoyo ligero de las puntas de los dedos en la pared, con los brazos separados del tronco y evitando que trasladasen el peso a través de los brazos a la pared, mientras el pie contralateral se situó ligeramente elevado del suelo. Se permitió un intento de elevación con el pie izquierdo, tras el cual se procedió a valorar la elevación del talón derecho. Se les pidió que elevaran el talón todo lo que pudieses al ritmo del metrónomo hasta que no pudieses elevar el talón por fatiga muscular. Si el sujeto compensaba con los brazos contra la pared o flexionaba la rodilla terminaba la prueba. También finalizaba si alcanzaba las 25 repeticiones, pues se ha establecido que estas son las repeticiones que de media se encuentran en la población sana ${ }^{23,24}$. Se anotó el número de repeticiones por cada pierna así como el grado de dificultad según la EEP.

La prueba de 6 minutos marcha (6MWT) se realizó previamente a la tercera sesión semanal de hemodiálisis, en el pasillo de la unidad. En el momento previo a la realización de la prueba se registró, mediante un esfigmomanómetro digital, la tensión arte- 
rial y la frecuencia cardiaca en el brazo sin fístula. A continuación se le indicó al paciente que durante 6 minutos, debía recorrer la máxima distancia señalada por unas marcas en el suelo, girando sin parar cada vez que recorría 20 metros. La orden dada al paciente fue la siguiente: $<<$ tiene que intentar recorrer la máxima distancia posible en este pasillo de 20 metros, andando lo más rápido que pueda sin llegar a correr $>$ >. Se permitió realizar el test con ayudas para la deambulación si el paciente las utilizaba en su vida diaria 0 con la asistencia de otra persona, o parar y reiniciar la marcha en caso de necesitar un descanso durante el test. Se registró la distancia recorrida y se pidió al paciente que describiera en la EEP cuál había sido el grado de dificultad de la prueba ${ }^{14,26}$.

\section{Análisis estadístico}

Los datos clínicos fueron analizados con la estadística descriptiva del SPSS versión 20.0 para Windows. El nivel de significación se predeterminó en $p \leq 0.05$ para todas los análisis estadísticos utilizados. Se presentan los resultados con la media (derivación estándar) si se acepta la normalidad de distribución o por la mediana (mínima y máximo). Se utilizó un análisis descriptivo para mostrar los datos de las historias clínicas más relevantes. El test de Shapiro Wilk se utilizó para ver la distribución de normalidad de las pruebas. Se ha empleado la prueba no paramétrica de Wilcoxon de muestras relacionadas para comparar las mediciones realizadas al inicio y al final del estudio cuando la muestra no se distribuía de forma normal, y la prueba $t$ de Student para muestras pareadas en caso de distribución normal.

\section{Resultados}

En la Tabla 1 podemos observar los datos descriptivos de las historias clínicas de la muestra más relevantes. La muestra analizada está compuesta por un total de 43 participantes de los cuales 28 eran hombres y 15 eran mujeres. La edad media de esta población es de $61.3 \pm 14.7$ años. La mediana de los meses que los pacientes llevan en hemodiálisis es de 49 meses siendo el valor mínimo de 18 meses y el valor máximo de 382 meses. La etiología más común de esta muestra era la glomerulonefritis (10 pacientes) seguida de la Diabetes Mellitus (9 pacientes).
En la Tabla 2 se describen los resultados de las pruebas realizadas en este estudio tanto al inicio como al final, representadas tanto con la media (DE) y la mediana (mínima-máximo).

El número de participantes disminuye en alguna de las pruebas. El motivo de éstas pérdidas son, la mayoría por fatiga u otros problemas de salud, a la hora de realizar las pruebas.

Como podemos observar en esta tabla, la mayoría de las pruebas de capacidad funcional que se completaron no muestran resultado significativos. Tres de las pruebas mejoran, de manera inesperada, significativamente: el STS 10 mejoró de 28.7 ( 10.6 - 60 segundos) a 25.5 (1.0 - 48.6 segundos); la dinamometría de la mano derecha incrementó de 25.7 (12.8) kg a 27.5 (14.5) kg; la dinamometría de la mano izquierda de 21.9 (12.4) $\mathrm{kg}$ a 24.6 (14.0) kg. Otras medidas no obtuvieron un deterioro significativo: SPPB, STS 60, elevación del talón izquierdo; 6MWT.

Respecto a los sujetos que abandonaron el estudio, la mitad tenían problemas serios de salud o fallecieron. 
[ Lucía Ortega Pérez de Villar, et al ]

Cuantificación del deterioro funcional durante seis meses en pacientes renales en estadio terminal

Tabla 2. Análisis estadístico que compara los resultados del inicio y el final del estudio.

\begin{tabular}{|c|c|c|c|c|c|c|c|}
\hline \multirow[b]{2}{*}{ Test } & \multirow{2}{*}{$\begin{array}{l}\text { N } \\
\text { Pre }\end{array}$} & \multirow[b]{2}{*}{ Post } & \multicolumn{2}{|l|}{ Pre } & \multicolumn{2}{|l|}{ Post } & \multirow[b]{2}{*}{$\mathbf{p}$} \\
\hline & & & $X(D E)$ & Mediana (min-max) & $X(D E)$ & Mediana (min-max) & \\
\hline SPPB* (0-12 puntos) & 42 & 42 & $10.02(3.03)$ & $12(4-12)$ & $10(2.89)$ & $11.50(2-12)$ & 0.0723 \\
\hline $\begin{array}{l}\text { Equilibrio monopodal } \\
\text { (máximo } 30 \text { segundos) }\end{array}$ & 43 & 42 & 10.25 (11.89) & $3.14(0-30)$ & $12.57(14.24)$ & $6.31(0-30)$ & 0.076 \\
\hline TUG* (segundos) & 43 & 43 & $9.30(4.01)$ & $8.12(0-21.66)$ & $9.15(4.69)$ & $8.16(0-25.84)$ & 0.134 \\
\hline STS $10 *$ (segundos) & 43 & 39 & $28.7(12.64)$ & $24.57(10.63-60)$ & $25.5(11.31)$ & $26.53(1.0-48.6)$ & 0.042 \\
\hline STS 60* (repeticiones) & 43 & 39 & 21.27 (9.05) & $21(2-48)$ & $19.32(9.4)$ & $20(0-37)$ & 0.224 \\
\hline Dinamometría Derecha $(\mathrm{kg})$ & 43 & 43 & $25.68(12.84)$ & $28(0-62)$ & $27.45(14.55)$ & $30(0-66)$ & 0.017 \\
\hline Dinamometría Izquierda (kg) & 43 & 43 & $21.90(12.39)$ & $22(1-58)$ & $24.55(14.01)$ & $28(0-65)$ & 0.015 \\
\hline $\begin{array}{l}\text { Elevación talón Derecho } \\
\text { (repeticiones) }\end{array}$ & 43 & 36 & $17.33(10.10)$ & $25(0-25)$ & $17.69(9.74)$ & $25(0-25)$ & 0.909 \\
\hline $\begin{array}{l}\text { Elevación talón Izquierdo } \\
\text { (repeticiones) }\end{array}$ & 43 & 36 & $16.05(10.19)$ & $22(0-25)$ & $16.81(9.36)$ & $19.5(0-25)$ & 0.306 \\
\hline 6MWT *(metros) & 42 & 34 & $390.94(131.42)$ & $396(84-678)$ & 383.63 (141.78) & $384(96-706)$ & 0.657 \\
\hline
\end{tabular}

\section{Discusión y conclusión}

La mayoría de los estudios de pacientes renales investigan cómo mejorar la calidad de vida relacionada con la salud de la población que sufre insuficiencia renal crónica con programas de ejercicio. Sin embargo, aún habiendo sido demostrada la eficacia del aumento de actividad física en este tipo de sujetos, son pocas las unidades de diálisis que ofrecen programas de ejercicios a sus pacientes. $Y$, como se ha descrito en diversos estudios, las personas con insuficiencia renal crónica en tratamiento de hemodiálisis se caracterizan por sufrir alteraciones en su sistema músculo-esquelético, como es la pérdida de fuerza y disminución de la masa muscular ${ }^{4,5,6}$, entre otro tipo de problemas, como alteraciones en el sistema cardiovascular y psicológicos. Lo que se ha intentado hacer en este estudio es describir cómo va disminuyendo el sistema músculo-esquelético en una población de pacientes que están en tratamiento de hemodiálisis desde hace años, y que la mayoría de ellos son personas sedentarias, en un periodo de tiempo de 6 meses. Se trata de una muestra de pacientes en hemodiálisis limitada y relativamente joven con un buen estado físico de salud. Llama la atención que, en ninguna de las pruebas de capacidad funcional que se ha realizado, se observa un deterioro significativo después de 6 meses. Incluso se ha visto una mejora en tres de las pruebas: en el STS-10 y dinamometría de mano derecha e izquierda. Los sujetos que no completaron los test eran personas con edad avanzada, que, después de 6 meses desde que comenzó el estudio, presentaban problemas serios de salud (problemas cardiacos e ingresos repetidos), lo que les impedía completar todas los test de capacidad funcional, además de ser los que peor condición física presentaban con respecto al resto de la muestra. Esto podría ser una de las causas por la que no se ha visto reflejada en la estadística el deterioro de capacidad funcional descrito por otros autores. $7,10,15$. Otras de las causas posibles de estos resultados puede ser la alta variabilidad en cuanto a la edad de la muestra. El sujeto de menor edad tenía 24 años y el de mayor edad 78 años. Cabe pensar que sujetos jóvenes van a tener mejor condición física y una vida más activa que las personas de edad avanzada. Al tratarse de una muestra relativamente joven podemos pensar que la mayoría de ellos son personas con una mejor condición física que las personas de mayor edad.

La principal limitación de este estudio es el pequeño tamaño de la muestra. Otra de las limitaciones que podemos haber encontrado es que la mayoría de los sujetos que participaron en el estudio son sujetos relativamente jóvenes que tienen una buena condición física, sin poder contar con las personas de edad avanzada que se encontraban en las unidades de diálisis. A pesar de estas limitaciones, debemos concienciar tanto a los profesionales sanitarios como a los propios pacientes la importancia de realizar un seguimiento de capacidad funcional ya que, como se ha descrito, a medida que los pacientes siguen con su tratamiento de hemodiálisis, se va produciendo un deterioro funcional entre otros, lo cual les produce tener una disminución de condición 
física y por ello de su calidad de vida relacionada con la salud.

Futuros estudios deberían incluir un mayor número de pacientes representativos a una muestra de pacientes con insuficiencia renal crónica y ampliar el periodo observacional.

En resumen, se recomienda a los centros de hemodiálisis realizar un seguimiento de capacidad funcional anualmente, ya que con un periodo de seis meses no se encuentran cambios significativos.

\section{Agradecimientos}

Agradecer al Servicio de Nefrología del Hospital Virgen del Consuelo de Valencia y Hospital Universitario Dr. Peset de Valencia su hospitalidad y colaboración para poder llevar a cabo este estudio, así como a todo el Departamento de Fisioterapia de la Universidad CEU Cardenal Herrera.

Recibido: 22 septiembre 2015

Revisado: 7 octubre 2015

Modificado: 8 noviembre 2015

Aceptado: 16 noviembre 2015

\section{Bibliografía}

1. ERA-EDTA Registry 2010, ERA-EDTA Registry Annual Report 2008, Academic Medical Center, Department of Medical Informatics, Amsterdam, The Nederlands.

2. ERA-EDTA Regsistry 2008, ERA-EDTA Registry Annual Report. 2006, Academic Medical Center, Department of Medical Informatics, Amsterdam, The Nederlands.

3. Johansen $\mathrm{KL}$, Chertow GM, Jin C, Kutner NG. Significance of frailty among dialysis patients. J Am Soc Nephrol 2007 Nov;18(11):2960-2967.

4. Brodin E, Ljungman S, Sunnerhagen KS. Rising from a chair: a simple screening test for physical function in predialysis patients. Scand J Urol Nephrol 2008;42(3):293-300.

5. Padilla J, Krasnoff J, Da Silva M, Hsu CY, Frassetto $\mathrm{L}$, Johansen $\mathrm{KL}$, et al. Physical functioning in patients with chronic kidney disease. J Nephrol 2008 JulAug;21(4):550-559.

6. Johansen $\mathrm{KL}$, Painter P, Kent-Braun JA, Ng AV, Carey $S$, Da Silva M, et al. Validation of questionnaires to estimate physical activity and functioning in end-stage renal disease. Kidney Int 2001 Mar;59(3):11211127.

7. Clegg A, Barber S, Young J, Forster A, Iliffe S. The Home-Based Older People's Exercise (HOPE) trial: study protocol for a randomised controlled trial. Trials 2011 Jun 8;12:143-6215-12-143.

8. Theou 0 , Jakobi JM, Vandervoort AA, Jones GR. A comparison of physical activity (PA) assessment tools across levels of frailty. Arch Gerontol Geriatr 2012 May-Jun;54(3):e307-14.

9. Chang YT, Wu HL, Guo HR, Cheng YY, Tseng CC, Wang MC, et al. Handgrip strength is an independent predictor of renal outcomes in patients with chronic kidney diseases. Nephrol Dial Transplant 2011 Nov;26(11):3588-3595.

10. Deligiannis A. Exercise rehabilitation and skeletal muscle benefits in hemodialysis patients. Clin Nephrol 2004 May;61 Suppl 1:S46-50.

11. Mhaolain AM, Gallagher D, Crosby L, Ryan D, Lacey $L$, Coen RF, et al. Frailty and quality of life for 
people with Alzheimer's dementia and mild cognitive impairment. Am J Alzheimers Dis Other Demen 2012 Feb;27(1):48-54.

12. Mitnitski A, Bao L, Skoog I, Rockwood K. A crossnational study of transitions in deficit counts in two birth cohorts: implications for modeling ageing. Exp Gerontol 2007 Mar;42(3):241-246.

13. Mitnitski A, Fallah N, Rockwood MR, Rockwood K. Transitions in cognitive status in relation to frailty in older adults: a comparison of three frailty measures. J Nutr Health Aging 2011 Dec;15(10):863-867.

14. Segura-Orti E, Rodilla-Alama V, Lison JF. Physiotherapy during hemodialysis: results of a progressive resistance-training programme. Nefrología 2008;28(1):67-72.

15. Álvarez-Ude, F., Fernández-Reyes, M.J., Vázquez, A., Mon, C., Sánchez, R. \& Rebollo, P. Physical symptoms and emotional disorders in patient on a periodic hemodialysis program. Nefrologia 2001; 21(2):191199.

16. Ostir GV, Ottenbacher KJ, Fried LP, Guralnik JM. The Effect of Depressive Symptoms on the Association Between Functional Status and Social Participation. Soc Indic Res 2007 Jan;80(2):379-392.

17. Hurvitz, E. A., Richardson, J. K., \& Werner, R. A. Unipodal stance testing in the assessment of peripheral neuropathy. Archives of Physical Medicine and Rehabilitation. 2001; 82(2), 198-204.

18. Podsiadlo D, Richardson S: The time "up \& go": a test of basic functional mobility for frail elderly persons. $J$ Am Geriatr Soc 1991, 39(2):142-148. 36.

19. Haweewannakij T, Wilaichit S, Chuchot R, Yuenyong Y, Saengsuwan J, Siritaratiwat W, et al. Reference values of physical performance in Thai elderly people who are functioning well and dwelling in the community. Phys Ther 2013 0ct;93(10):1312-1320.

20. Silva LF, Matos CM, Lopes GB, Martins MT, Martins MS, Arias LU, et al. Handgrip strength as a simple indicator of possible malnutrition and inflammation in men and women on maintenance hemodialysis. J Ren Nutr 2011 May;21(3):235-245.

21. Leal V0, Mafra D, Fouque D, Anjos LA. Use of handgrip strength in the assessment of the muscle function of chronic kidney disease patients on dialysis: a systematic review. Nephrol Dial Transplant 2011 Apr;26(4):1354-1360.

22. Csuka M, McCarty DJ. Simple method for measurement of lower extremity muscle strength. Am J Med. 1985;78:77-81.

23. Svantesson U, Osterberg U, Thomee R, Grimby G. Muscle fatigue in a standing heel-rise test. Scand $J$ Rehabil Med. 1998; 30:67-72.

24. Portney LG, Watkins MP. Foundations of Clinical Research: Applications to Practice. 2nd ed. Upper Saddle River, NJ: Prentice Hall Health; 2000.

25. Sekir U, Yildiz Y, Hazneci B, et al. Reliability of a functional test battery evaluating functionality, proprioception, and strength in recreational athletes with functional ankle instability. Eur J Phys Rehabil Med. 2008;44:407-415.

26. ATS Committee on Proficiency Standards for Clinical Pulmonary Function Laboratories. ATS statement: guidelines for the six minute walk test. Am J Respir Crit Care Med. 2002;166:1 11-117.

\section{ANEXOS}

\section{ESCALA DE ESFUERZO PERCIBIDO (EEP)} ¿Cómo nota el ejercicio?

\begin{tabular}{|l|l|}
\hline 6 & - \\
\hline 7 & Muy, muy suave \\
\hline 8 & - \\
\hline 9 & Muy suave \\
\hline 10 & - \\
\hline 11 & Ligero \\
\hline 12 & - \\
\hline 13 & Algo duro \\
\hline 14 & - \\
\hline 15 & Duro \\
\hline 16 & - \\
\hline 17 & Muy duro \\
\hline 18 & - \\
\hline 19 & Muy, muy duro \\
\hline 20 & Máximo esfuerzo percibido \\
\hline
\end{tabular}

Figura 1. Escala de Esfuerzo Percibido. (EEP) 